Open Access

\title{
The generalized Cauchy family of distributions with applications
}

\author{
Ayman Alzaatreh ${ }^{1 *}$, Carl Lee ${ }^{2}$, Felix Famoye ${ }^{2}$ and Indranil Ghosh ${ }^{3}$
}

\author{
* Correspondence: \\ ayman.alzaatreh@nu.edu.kz \\ 'Department of Mathematics, \\ Nazarbayev University, Astana, KZ \\ 010000, Kazakhstan \\ Full list of author information is \\ available at the end of the article
}

\begin{abstract}
A family of generalized Cauchy distributions, T-Cauchy\{Y\} family, is proposed using the $T-R\{Y\}$ framework. The family of distributions is generated using the quantile functions of uniform, exponential, log-logistic, logistic, extreme value, and Fréchet distributions. Several general properties of the T-Cauchy\{Y\} family are studied in detail including moments, mean deviations and Shannon's entropy. Some members of the $T$-Cauchy $\{Y\}$ family are developed and one member, gamma-Cauchy\{exponential\} distribution, is studied in detail. The distributions in the $T$-Cauchy\{$\{Y\}$ family are very flexible due to their various shapes. The distributions can be symmetric, skewed to the right or skewed to the left.

Keywords: T-R\{Y\} framework, Quantile function, Moments, Shannon's entropy
\end{abstract}

\section{Introduction}

The Cauchy distribution, named after Augustin Cauchy, is a simple family of distributions for which the expected value does not exist. Also, the family is closed under the formation of sums of independent random variables, and hence is an infinitely divisible family of distributions. The Cauchy distribution was used by Stigler (1989) to obtain an explicit expression for $P\left(Z_{1} \leq 0, Z_{2} \leq 0\right)$ where $\left(Z_{1}, Z_{2}\right)^{T}$ follows the standard bivariate normal distribution. The Cauchy distribution has been used in many applications such as mechanical and electrical theory, physical anthropology, measurement problems, risk and financial analysis. It was also used to model the points of impact of a fixed straight line of particles emitted from a point source (Johnson et al. 1994). In Physics, it is called a Lorenzian distribution, where it is the distribution of the energy of an unstable state in quantum mechanics.

Eugene et al. (2002) introduced the beta-generated family of distributions using the beta as the baseline distribution. Based on the beta-generated family, Alshawarbeh et al. (2013) proposed the beta-Cauchy distribution. The beta-generated family was extended by Alzaatreh et al. (2013) to the $T-R(W)$ family. The cumulative distribution function (CDF) of the $T-R(W)$ distribution is $G(x)=\int_{a}^{W(F(x))} r(t) d t$, where $r(t)$ is the probability density function (PDF) of a random variable $T$ with support $(a, b)$ for $-\infty \leq a<b \leq \infty$. The link function $W:[0,1] \rightarrow \mathbb{R}$ is monotonic and absolutely continuous with $W(0) \rightarrow a$ and $W(1) \rightarrow b$.

(c) 2016 The Author(s). Open Access This article is distributed under the terms of the Creative Commons Attribution 4.0 International License (http://creativecommons.org/licenses/by/4.0/), which permits unrestricted use, distribution, and reproduction in any medium, provided you give appropriate credit to the original author(s) and the source, provide a link to the Creative Commons license, and indicate if changes were made. 
Aljarrah et al. (2014) considered the function $W($.) to be the quantile function of a random variable $Y$ and defined the $T-R\{Y\}$ family. In the $T-R\{Y\}$ framework, the random variable $T$ is a 'transformer' that is used to 'transform' the random variable $R$ into a new family of generalized distributions of $R$. Many families of generalized distributions have appeared in the literature. Alzaatreh et al. $(2014,2015)$ studied the $T$-gamma and the $T$-normal families. Almheidat et al. (2015) studied the $T$ Weibull family. In this paper, a family of generalized Cauchy distribution is proposed and studied.

This article focuses on the generalization of the Cauchy distribution and studies some new distributions and their applications. The article gives a brief review of the $T-R\{Y\}$ framework and defines several new generalized Cauchy sub-families. It contains some general properties of the $T$-Cauchy $\{Y\}$ distributions. A member of the $T$-Cauchy $\{Y\}$ family, the gamma-Cauchy\{exponential\} distribution, is studied. The study includes moments, estimation and applications. Some concluding remarks were provided.

\section{The $T$-Cauchy $\{\eta\}$ family of distributions}

The $T-R\{Y\}$ framework defined in Aljarrah et al. (2014) (see also Alzaatreh et al. $2014)$ is given as follows. Let $T, R$ and $Y$ be random variables with $\operatorname{CDF} F_{Z}(x)=$ $P(Z \leq x)$, and corresponding quantile function $Q_{Z}(p)$, where $Z=T, R, Y$ and the quantile function is defined as $Q_{Z}(p)=\inf \left\{z: F_{Z}(z) \geq p\right\}, 0<p<1$. If densities exist, we denote them by $f_{Z}(x)$, for $Z=T, R$ and $Y$. Now assume the random variables $T, Y \in(a, b)$ for $-\infty \leq a<b \leq \infty$. The random variable $X$ in $T-R\{Y\}$ family of distributions is defined as

$$
F_{X}(x)=\int_{a}^{Q_{Y}\left(F_{R}(x)\right)} f_{T}(t) d t=F_{T}\left(Q_{Y}\left(F_{R}(x)\right)\right) .
$$

The corresponding PDF associated with (1) is

$$
f_{X}(x)=f_{T}\left(Q_{Y}\left(F_{R}(x)\right)\right) \times Q_{Y}^{\prime}\left(F_{R}(x)\right) \times f_{R}(x) .
$$

Alternatively, (2) can be written as

$$
f_{X}(x)=f_{R}(x) \times \frac{f_{T}\left(Q_{Y}\left(F_{R}(x)\right)\right)}{f_{Y}\left(Q_{Y}\left(F_{R}(x)\right)\right)} .
$$

The hazard function of the random variable $X$ can be written as

$$
h_{X}(x)=h_{R}(x) \times \frac{h_{T}\left(Q_{Y}\left(F_{R}(x)\right)\right)}{h_{Y}\left(Q_{Y}\left(F_{R}(x)\right)\right)} .
$$

Alzaatreh et al. (2013, 2014, 2015) studied, respectively, the $T$-R $\{$ exponential\}, $T$-normal $\{Y\}$ and $T$-gamma $\{Y\}$ families of distributions. Aljarrah et al. (2014) studied some general properties of the $T-R\{Y\}$ family. Next, we define the $T$-Cauchy $\{Y\}$ family. 
Let $R$ be a random variable that follows the Cauchy distribution with $\operatorname{PDF} f_{R}(x)=$ $f_{C}(x)=\pi^{-1} \theta^{-1}\left(1+(x / \theta)^{2}\right)^{-1}$ and $\operatorname{CDF} F_{R}(x)=F_{C}(x)=0.5+\pi^{-1} \tan ^{-1}(x / \theta), x \in \mathbb{R}, \theta>0$, then (3) reduces to

$$
f_{X}(x)=f_{C}(x) \times \frac{f_{T}\left(Q_{Y}\left(F_{C}(x)\right)\right)}{f_{Y}\left(Q_{Y}\left(F_{C}(x)\right)\right)} .
$$

Hereafter, the family of distributions in (5) will be called the $T$-Cauchy $\{Y\}$ family. It is clear that the PDF in (5) is a generalization of Cauchy distribution. From (1), if $T \stackrel{d}{=} Y$, then $X \stackrel{d}{=} \operatorname{Cauchy}(\theta)$. Also, if $Y \stackrel{d}{=} \operatorname{Cauchy}(\theta)$, then $X \stackrel{d}{=} T$. Furthermore, when $T \sim \operatorname{beta}(a, b)$ and $Y \sim \operatorname{uniform}(0,1)$, the $T$-Cauchy $\{Y\}$ reduces to the betaCauchy distribution (Alshawarbeh et al. 2013). When $T \sim \operatorname{Power}(a)$ and $Y \sim$ uniform(0, 1), the $T$-Cauchy $\{Y\}$ reduces to the exponentiated-Cauchy distribution (Sarabia and Castillo 2005). Table 1 gives six quantile functions of known distributions (in standard form) which will be applied to generate $T$-Cauchy $\{Y\}$ sub-families in the following subsections. It is straightforward to use non-standard quantile functions. By using non-standard quantile functions, many resulting distributions in the $T-R\{Y\}$ family will have more than five parameters, which are not practically useful (Johnson et al. 1994, p. 12). Thus, we focus on the standard quantile functions in this paper.

\subsection{T-Cauchy\{uniform\} family of distributions}

By using the quantile function of the uniform distribution in Table 1, the corresponding CDF to (1) is

$$
F_{X}(x)=F_{T}\left\{F_{C}(x)\right\},
$$

and the corresponding PDF to (6) is

$$
f_{X}(x)=f_{C}(x) \times f_{T}\left(F_{C}(x)\right), x \in \mathbb{R} .
$$

\subsection{T-Cauchy\{exponential\} family of distributions}

By using the quantile function of the exponential distribution in Table 1, the corresponding CDF to (1) is

$$
F_{X}(x)=F_{T}\left\{-\log \left(1-F_{C}(x)\right)\right\}
$$

and the corresponding PDF to (8) is

$$
f_{X}(x)=\frac{f_{C}(x)}{1-F_{c}(x)} \times f_{T}\left(-\log \left(1-F_{c}(x)\right)\right), x \in \mathbb{R} .
$$

Table 1 Quantile functions for different $Y$ distributions

\begin{tabular}{ll}
\hline$Y$ & $Q_{Y}(p)$ \\
\hline (a) Uniform & $p$ \\
(b) Exponential & $-\log (1-p)$ \\
(c) Log-logistic & $p /(1-p)$ \\
(d) Logistic & $\log [p /(1-p)]$ \\
(e) Extreme value & $\log [-\log (1-p)]$ \\
(f) Fréchet & $-1 / \log (1-p)$ \\
\hline
\end{tabular}


Note that the CDF and the PDF in (8) and (9) can be written as $F_{X}(x)=$ $F_{T}\left(H_{C}(x)\right)$ and $f_{X}(x)=h_{C}(x) f_{T}\left(H_{C}(x)\right)$ where $h_{C}(x)$ and $H_{C}(x)$ are the hazard and cumulative hazard functions for the Cauchy distribution, respectively. Therefore, the $T$-Cauchy\{exponential\} family of distributions arises from the 'hazard function of the Cauchy distribution'.

\subsection{T-Cauchy\{log-logistic\} family of distributions}

By using the quantile function of the log-logistic distribution in Table 1 , the corresponding CDF to (1) is

$$
F_{X}(x)=F_{T}\left\{F_{C}(x) /\left[1-F_{C}(x)\right]\right\}
$$

and the corresponding PDF is

$$
f_{X}(x)=\frac{f_{C}(x)}{\left(1-F_{C}(x)\right)^{2}} \times f_{T}\left(\frac{F_{C}(x)}{1-F_{C}(x)}\right), x \in \mathbb{R} .
$$

which is a family of generalized Cauchy distributions arising from the 'odds' of the Cauchy distribution.

\subsection{T-Cauchy\{logistic\} family of distributions}

By using the quantile function of the logistic distribution in Table 1, the corresponding CDF to (1) is

$$
F_{X}(x)=F_{T}\left\{\log \left(F_{C}(x) /\left[1-F_{C}(x)\right]\right)\right\}
$$

and the corresponding PDF is

$$
f_{X}(x)=\frac{f_{C}(x)}{F_{C}(x)\left[1-F_{C}(x)\right]} \times f_{T}\left(\log \left(F_{C}(x) /\left[1-F_{C}(x)\right]\right)\right), x \in \mathbb{R} .
$$

Note that (13) can be written as $f_{X}(x)=\frac{h_{C}(x)}{F_{C}(x)} \times f_{T}\left(\log \left(\frac{F_{C}(x)}{1-F_{C}(x)}\right)\right)$, which is a family of generalized Cauchy distributions arising from the 'logit function' of the Cauchy distribution.

\subsection{T-Cauchy\{extreme value\} family of distributions}

By using the quantile function of the extreme value distribution in Table 1, the corresponding CDF to (1) is

$$
F_{X}(x)=F_{T}\left\{\log \left(-\log \left[1-F_{C}(x)\right]\right)\right\},
$$

and the corresponding PDF is

$$
f_{X}(x)=\frac{f_{C}(x)}{\left[F_{C}(x)-1\right] \log \left(1-F_{C}(x)\right)} \times f_{T}\left\{\log \left[-\log \left(1-F_{C}(x)\right)\right]\right\}, x \in \mathbb{R} .
$$

The CDF in (14) and the PDF in (15) can be written as $F_{x}(x)=F_{T}\left(\log H_{C}(x)\right)$ and $f_{X}(x)=\left\{h_{C}(x) / H_{C}(x)\right\} f_{T}\left(\log H_{C}(x)\right)$ respectively. 


\subsection{T-Cauchy\{Fréchet $\}$ family of distributions}

By using the quantile function of the Fréchet distribution in Table 1, the corresponding CDF to (1) is

$$
F_{X}(x)=F_{T}\left\{-1 / \log \left(F_{C}(x)\right)\right\},
$$

and the corresponding PDF is

$$
f_{X}(x)=\frac{f_{C}(x)}{F_{C}(x)\left(\log F_{C}(x)\right)^{2}} \times f_{T}\left\{-1 / \log \left(F_{C}(x)\right)\right\}, x \in \mathbb{R} .
$$

Figures 1 and 2 show some examples of two members of the $T$-Cauchy $\{Y\}$ family. The first example is Weibull-Cauchy\{exponential\} distribution which can be obtained by replacing the random variable $T$ in (9) with Weibull $(c, \gamma)$ random variable. The second example is Lomax-Cauchy\{log-logistic\} distribution which can be obtained by replacing the random variable $T$ in (11) with $\operatorname{Lomax}(\alpha, \lambda)$ random variable. From the figures, it appears that the shapes of the distributions can be left-skewed, rightskewed or symmetric.

\section{Some properties of the $T$-Cauchy $\{Y\}$ family of distributions}

In this section, we discuss some general properties of the $T$-Cauchy family of distributions. The proofs are omitted for straightforward results.

Lemma 1: Let $T$ be a random variable with $\operatorname{PDF} f_{T}(x)$, then

(i) The random variable $X=-\theta \cot \left(\pi F_{Y}(T)\right)$ follows the $T$-Cauchy $\{Y\}$ distribution.

(ii) The quantile function for $T$-Cauchy $\{Y\}$ family is $Q_{X}(p)=-\theta \cot \left(\pi F_{Y}\left(Q_{T}(p)\right)\right)$.

The Shannon's entropy (Shannon 1948) of a random variable $X$ is a measure of variation of uncertainty and it is defined as $\eta_{X}=-E\{\log (f(X))\}$. The following proposition provides an expression for the Shannon's entropy for the $T$-Cauchy $\{Y\}$ family.

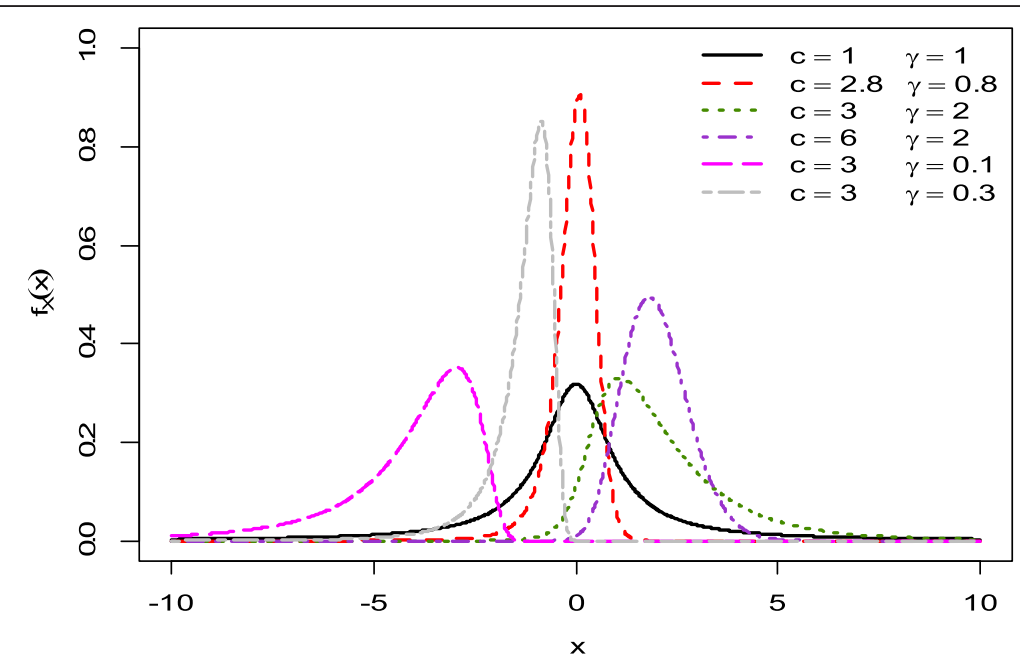

Fig. 1 Graphs for the PDF of the Weibull-Cauchy\{exponential\} distribution when $\theta=1$ 

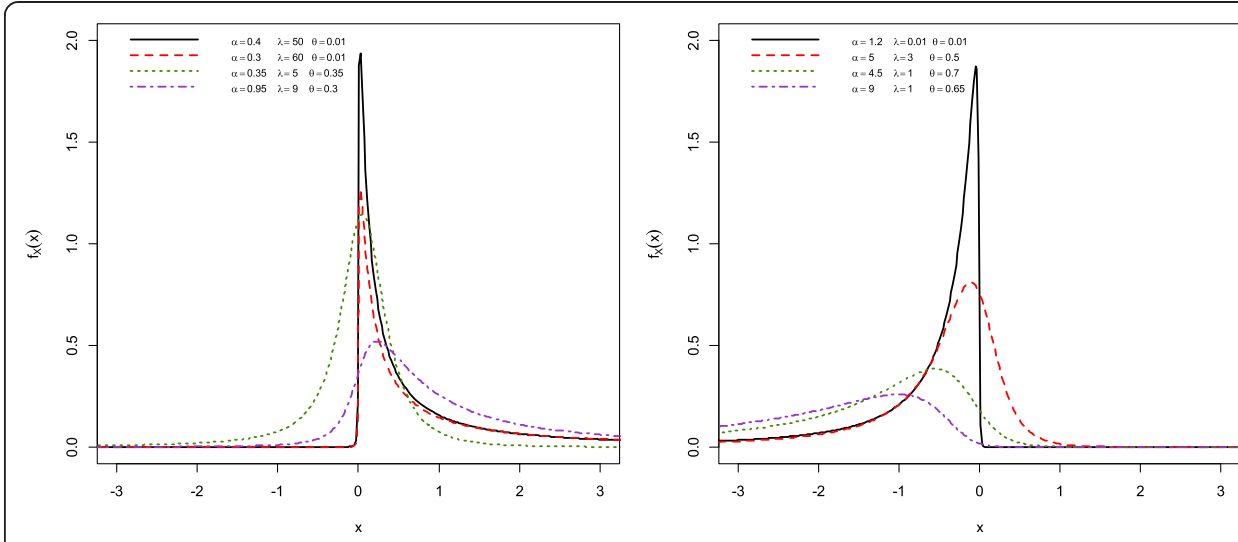

Fig. 2 Graphs for the PDF of the Lomax-Cauchy\{log-logistic\} distribution

Proposition 1: The Shannon's entropy for the T-Cauchy $\{Y\}$ family is given by

$$
\eta_{X}=\log (\theta)-\log (\pi)+\eta_{T}+E\left(\log f_{Y}(T)\right)-2 E\left\{\log \left(F_{Y}(T)\right)\right\}-2 \sum_{j=1}^{\infty} V_{j} E\left[F_{Y}(T)\right]^{2 j}
$$

Proof: By using the result in Aljarrah et al. (2014), the Shannon's entropy for the $T$-Cauchy $\{Y\}$ is

$$
\eta_{X}=\eta_{T}+E\left(\log f_{Y}(T)\right)-E\left\{\log f_{C}\left(Q_{C}\left(F_{Y}(T)\right)\right)\right\}
$$

Now, one can show that

$$
\log f_{C}\left(Q_{C}\left(F_{Y}(t)\right)\right)=-\log (\pi \theta)+2 \log \left(\sin \left(\pi F_{Y}(t)\right)\right) .
$$

On using the following series expansion from Gradshteyn and Ryzhik (2007, p. 55)

$$
\log (\sin (\pi x))=\log (\pi x)+\sum_{j=1}^{\infty} V_{j} x^{2 j}
$$

where $\frac{V_{j}=(-1)^{j}(2 \pi)^{2 j} B_{2 j}}{2 j(2 j) !}$ and $B_{j}$ is the Bernoulli number, we get the result in (18).

Next proposition gives a general expression for the $r$-th moment for the $T$-Cauchy $\{Y\}$ family.

Proposition 2: The $r$-th moment for the $T$-Cauchy $\{Y\}$ family of distributions is given by

$$
E\left(X^{r}\right)=(-1)^{r} \theta^{r} \sum_{k=0}^{\infty} c_{k} E\left[F_{Y}(T)\right]^{2 k-r},
$$

where $c_{0}=\pi^{-r}, c_{m}=\pi m^{-1} \sum_{k=1}^{m}(k r-m+k) w_{k} c_{m-k}, m \geq 1$ and $w_{k}=\frac{(-1)^{k} 2^{2 k} B_{2 k} \pi^{2 k-1}}{(2 k) !}$.

Proof: From Lemma 1(i), the $r$-th moment for the T-Cauchy $\{Y\}$ family can be written as $E\left(X^{r}\right)=(-1)^{r} \theta^{r} E\left(\cot \pi\left(F_{Y}(T)\right)\right)^{r}$. Now, using the following series expansion (see Abramowitz and Stegun 1964, p.75), $\cot (\pi x)=\sum_{k=0}^{\infty} w_{k} x^{2 k-1},|x|<\pi$, where $w_{k}=\frac{(-1)^{k} 2^{2 k} B_{2 k} \pi^{2 k-1}}{(2 k) !}$. Therefore,

$$
\left(\cot \pi\left(F_{Y}(t)\right)\right)^{r}=\sum_{k=0}^{\infty} c_{k}\left(F_{Y}(t)\right)^{2 k-r},
$$

where $c_{0}=\pi^{-r}, \quad c_{m}=\pi m^{-1} \sum_{k=1}^{m}(k r-m+k) w_{k} c_{m-k}, m \geq 1 \quad$ [see Gradshteyn and Ryzhik
2007, p. 17]. 
As an example of the applicability of the results in Lemma 1 and Propositions 1 and 2 , we use these results and apply them on the $T$-Cauchy\{exponential\}. One can get similar results by choosing any of the $T$-Cauchy $\{Y\}$ families.

Corollary 1: Based on Lemma 1, if $T$ is a random variable with $\operatorname{PDF} f_{T}(x)$, then

(i) The random variable $X=\theta \cot \left(\pi e^{-T}\right)$ follows a distribution in the $T$ -

Cauchy\{exponential\} family.

(ii) The quantile functions for $T$-Cauchy\{exponential\} family is $Q_{X}(p)=\theta \cot \left(\pi e^{-Q_{T}(p)}\right)$.

Corollary 2: The Shannon's entropy for the T-Cauchy\{exponential\} family is given by

$$
\eta_{X}=\log (\theta)-\log (\pi)+\eta_{T}+\mu_{T}-2 \sum_{j=1}^{\infty} V_{j} M_{T}(-2 j)
$$

where $M_{T}($.$) is the moment generating function of the random variable T$.

Proof: The result follows from Proposition 1 and the fact that $E\left(\log f_{Y}(T)\right)=\mu_{T}$

Corollary 3: The $r$-th moment for the $T$-Cauchy\{exponential\} family of distributions is given by

$$
E\left(X^{r}\right)=\theta^{r} \sum_{k=0}^{\infty} c_{k} M_{T}(r-2 k),
$$

where $c_{k}$ is defined in Proposition 2.

Proof: The result follows from Proposition 2 and the fact that $\cot \left(\pi F_{Y}(u)\right)=-\cot \left(\pi e^{-u}\right)$.

Proposition 3: The mode(s) of the T-Cauchy\{exponential\} family are the solutions of the equation

$$
x=\frac{\theta}{2 \pi} h_{C}(x)\left\{1+\frac{f_{T}^{\prime}\left(H_{C}(x)\right)}{f_{T}\left(H_{C}(x)\right)}\right\} .
$$

Proof: For Cauchy distribution, one can show that $f_{C}^{\prime}(x)=-2 \pi \theta^{-1} x f_{C}^{2}(x)$ and $h_{C}^{\prime}(x)=-2 \pi \theta^{-1} x h_{C}(x)+h_{C}^{2}(x)$. On finding $f_{X}^{\prime}(x)$ by using Eq. (9) and setting the derivative to zero, it is easy to get the result in (24).

\section{Gamma-Cauchy\{exponential\} distribution}

For the remaining sections, we investigate in details the properties, parameter estimation and applications of a new distribution of the $T$-Cauchy $\{Y\}$ family, the gammaCauchy\{exponential\} distribution. This distribution is interesting as it consists of special cases of exponentiated Cauchy and distributions of record values from the Cauchy distribution. Let $T$ be a random variable that follows the gamma distribution with parameters $\alpha$ and $\beta$. From Eqs. (8) and (9), the PDF and CDF of gamma-Cauchy\{exponential\} distribution are, respectively, given by

$$
\begin{aligned}
& f(x)=\frac{\left[-\log \left(0.5-\pi^{-1} \tan ^{-1}(x / \theta)\right)\right]^{\alpha-1}\left[0.5-\pi^{-1} \tan ^{-1}(x / \theta)\right]^{\frac{1}{\beta}-1}}{\pi \theta \beta^{\alpha} \Gamma(\alpha)\left(1+(x / \theta)^{2}\right)}, x \in \mathbb{R}, \\
& F(x)=\frac{\gamma\left[\alpha,-\beta^{-1} \log \left(0.5-\pi^{-1} \tan ^{-1}(x / \theta)\right)\right]}{\Gamma(\alpha)}, x \in \mathbb{R},
\end{aligned}
$$


where $\gamma(\alpha, x)=\int_{0}^{x} t^{\alpha-1} e^{-t} d t$ is the incomplete gamma function. For simplicity, a random variable $X$ with PDF $f(x)$ in (25) is said to follow the gamma-Cauchy\{exponential\} distribution and is denoted by $G C(\alpha, \beta, \theta)$.

Some special cases are worth mentioning:

(i) $G C(1, \beta, \theta)$ is the exponentiated Cauchy distribution proposed by Sarabia and Castillo (2005). In particular $G C(1,1,1)$ is the standard Cauchy distribution.

(ii) $G C\left(1, n^{-1}, \theta\right), n \in \mathbb{N}$ is the distribution of the minimum of $n$ independent Cauchy random variables.

(iii) $G C(n+1,1, \theta), n \in \mathbb{N}$ is the distribution of the $n$th upper record in a sequence of independent Cauchy random variables.

Remarks: The following results follow from Corollary 1, Corollary 2 and Proposition 3.

(i) If a random variable $Y$ follows a gamma distribution with parameters $\alpha$ and $\beta$, then $X=\theta \cot \left(\pi e^{-Y}\right)$ follows the $G C(\alpha, \beta, \theta)$ distribution.

(ii) The quantile function of $G C(\alpha, \beta, \theta)$ is $Q(p)=\theta \cot \left(\pi e^{-\beta^{\gamma^{-1}}[\alpha, p \Gamma(\alpha)]}\right), \quad 0<p<1$.

(iii) The Shannon's entropy for the $G C(\alpha, \beta, \theta)$ distribution is given by

$$
\eta_{X}=\alpha(1+\beta)+\log \left(\pi^{-1} \theta \beta \Gamma(\alpha)\right)+(1-\alpha) \psi(\alpha)-2 \sum_{j=1}^{\infty} V_{j}(1+2 j \beta)^{-\alpha} \text {, where } \psi(.) \text { is the }
$$

digamma function and $V_{j}$ is defined in Eq. (21).

Proposition 4: The $G C(\alpha, \beta, \theta)$ distribution is unimodal and the mode is at $m=\theta x$ where $x$ is the solution of the equation

$$
k(x)=\log \left(\frac{\cot ^{-1}(x)}{\pi}\right)\left[2 x \cot ^{-1}(x)-1+1 / \beta\right]+\alpha-1=0 .
$$

Proof: It is not difficult to show that the mode of $f(x)$ in (25) is the solution of $k(x / \theta)=0$, where $k(x)$ is defined above. Therefore, the mode of $f(x)$ is at $m=\theta x$ where $k(x)=0$. To show the unimodality of $f(x)$, consider $A(x)=\log \left(\pi^{-1} \cot ^{-1}(x)\right)$ and $B(x)=2 x \cot ^{-1}(x)$. Clearly $A(x)$ is a strictly decreasing function (since it is equal to $\left.\log \left(1-F_{C}(x)\right)\right)$. Furthermore, $A(x)<0$ for all $x \in \mathbb{R}$. Now, $B^{\prime}(x)=2[-x /(1+$ $\left.\left.x^{2}\right)+\cot ^{-1}(x)\right]$. Therefore, $B^{\prime}(x)>0$ for all $x \leq 0$. If $x>0$, we have $B^{\prime}(x)<B^{\prime}(0)=\pi / 2$ since $B^{\prime \prime}(x)<0$. Since $\lim _{x \rightarrow \infty} B^{\prime}(x)=0$. we get $B^{\prime}(x)>0$ for all $x>0$. Therefore, $B(x)$ is strictly increasing for all $x \in \mathbb{R}$. Now, let us prove the claim that $\eta(x)=A(x) B(x)$ is a decreasing function on $\mathbb{R}$.

Proof of the claim: Let $0 \leq x \leq y$, then $0 \leq-A(x) \leq-A(y)$ and $0 \leq B(x) \leq B(y)$. This implies that $\eta(x) \geq \eta(y)$. Now let $x<0$, then $\eta^{\prime}(x)=-2 x /\left(x^{2}+1\right)-2\left(x^{2}+1\right)^{-1} x$ $\log \left(\pi^{-1} \cot ^{-1}(x)\right)+2 \cot ^{-1}(x) \log \left(\pi^{-1} \cot ^{-1}(x)\right)$. Since the middle term in $\eta^{\prime}(x)$ is negative, consider $\quad \psi(x)=\frac{x}{x^{2}+1}-\cot ^{-1}(x) \log \left(\cot ^{-1}(x) / \pi\right)$. On differentiation, $\quad \psi^{\prime}(x)=\frac{1}{x^{2}+1}$ $\left\{\frac{2}{x^{2}+1}+\log \left(\cot ^{-1}(x) / \pi\right)\right\}$. It is easy to show that the term $\zeta(x)=\frac{2}{x^{2}+1}+\log$ $\left(\cot ^{-1}(x) / \pi\right)$ is strictly increasing on $x \leq 0$ with $\zeta(0)>0$ and $\zeta(-\infty) \rightarrow 0$. Thus, $\zeta(x)>0$ for all $x<0$. This implies that $\psi(x)$ is strictly increasing on $x<0$ with $\psi(0)>0$ and $\psi(-\infty) \rightarrow 0$. That is, $\psi(x)>0$ for all $x<0$. Therefore $\eta^{\prime}(x) \leq 0$ for all $x<0$. 
Hence, $\eta(x)=A(x) B(x)$ is a decreasing function in $\mathbb{R}$. This completes the proof of the claim. The fact that $\eta(-\infty) \rightarrow 2$ and $\eta(\infty) \rightarrow-\infty$ implies that $\eta(x)=0$ has a unique solution. Now, $B(x)-1+1 / \beta$ is only a shift by $-1+1 / \beta$ and therefore remains a strictly increasing function. One can show that the term $A(x)[B(x)-1+1 / \beta]$ remains a decreasing function for all $x \in \mathbb{R}$ and hence $k(x)$ remains a decreasing function in $\mathbb{R}$ with $k(-\infty) \rightarrow \alpha+1>0$ and $k(\infty) \rightarrow-\infty$. This ends the proof.

In Fig. 3, various graphs of $f(x)$ are provided for different parameter values of $\alpha$ and $\beta$ where $\theta=1$. The plots indicate that the gamma-Cauchy\{exponential\} distribution can be symmetric, right-skewed or left-skewed. Also, it appears that gammaCauchy\{exponential\} is symmetric only for the trivial case when $\alpha=\beta=1$.

In the following subsection, we provide some results related to the moments of $G C(\alpha, \beta, \theta)$ distribution.

\subsection{Moments of gamma-Cauchy\{exponential\} distribution}

From Corollary 3 , the $r$-th moment of the $G C(\alpha, \beta, \theta)$ can be written as

$$
\mu_{r}^{\prime}(\alpha, \beta, \theta)=E\left(X^{r}\right)=\theta^{r} \sum_{k=0}^{\infty} c_{k}[1-\beta(r-2 k)]^{-\alpha},
$$

where $c_{k}$ is defined in Eq. (22). Therefore, the mean of $G C(\alpha, \beta, \theta)$ is

$$
\mu_{1}^{\prime}(\alpha, \beta, \theta)=\theta \sum_{k=0}^{\infty} c_{k}[1-\beta(1-2 k)]^{-\alpha}
$$

where $c_{k}$ is defined in (22) with $r=1$. Note that $\mu_{1}^{\prime}(\alpha, \beta, \theta)$ is defined here for $\alpha>1$ and $\beta<1$.

The next proposition establishes the condition for the existence of $r$-th moment of the $G C(\alpha, \beta, \theta)$ distribution.

Proposition 5: The $r$-th moment of the $G C(\alpha, \beta, \theta)$ distribution exists if and only if $\alpha>r$ and $\beta^{-1}>r$.

Proof: Without loss of generality, we assume $\theta=1$ and apply a similar idea as in Alshawarbeh et al. (2012). We write

$$
E\left(X^{r}\right)=\int_{-\infty}^{-1} x^{r} g(x) d x+\int_{-1}^{1} x^{r} g(x) d x+\int_{1}^{\infty} x^{r} g(x) d x .
$$
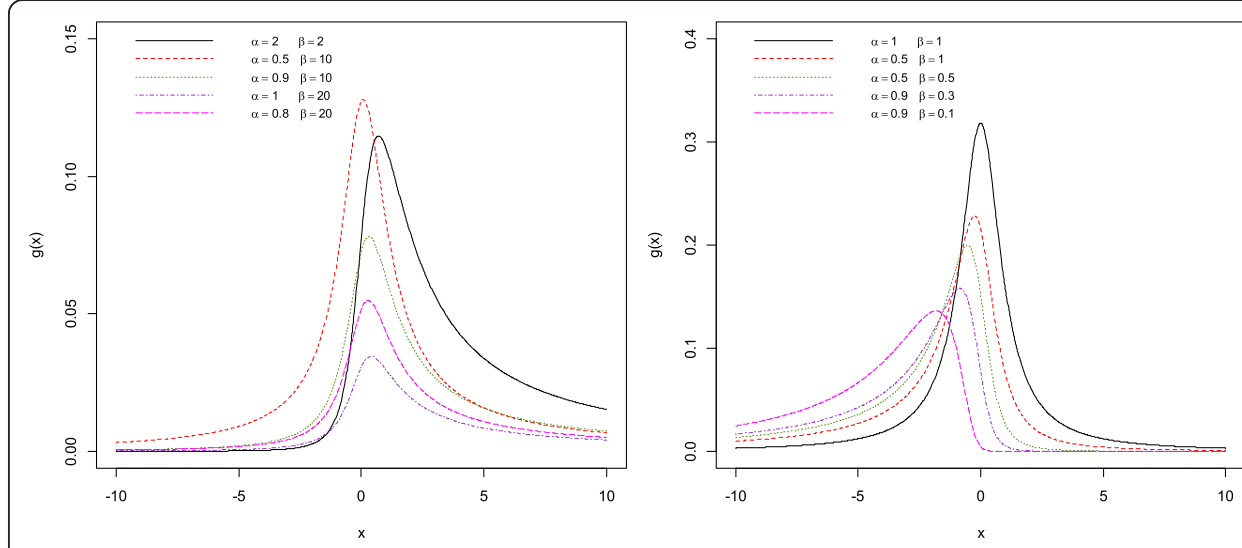

Fig. 3 Graphs for the PDF of the gamma-Cauchy\{exponential\} distribution when $\theta=1$ 
Since the middle integrand is bounded by 2 , it suffices to investigate the existence of the first and third integrands of the right hand side of Eq. (28). Consider $I_{1}=\int_{1}^{\infty} x^{r} g(x) d x$ and $I_{2}=\int_{-\infty}^{-1} x^{r} g(x) d x$. Consider the following inequality from Abramowitz and Stegun (1964), p. 68

$$
x<-\log (1-x)<\frac{x}{1-x}, \quad x<1, x \neq 0 .
$$

On using the inequality in (29) and for $\alpha>1$, we have

$$
I_{1} \leq \frac{1}{\pi \beta^{\alpha} \Gamma(\alpha)} \int_{1}^{\infty} \frac{x^{r}}{1+x^{2}}\left(1 / 2+\pi^{-1} \tan ^{-1}(x)\right)^{\alpha-1}\left(1 / 2-\pi^{-1} \tan ^{-1}(x)\right)^{1 / \beta-\alpha} d x
$$

Let us write $\delta(x)=\frac{x^{r}}{1+x^{2}}\left(1 / 2+\pi^{-1} \tan ^{-1}(x)\right)^{\alpha-1}\left(1 / 2-\pi^{-1} \tan ^{-1}(x)\right)^{1 / \beta-\alpha}$. Then one can show that as $x \rightarrow \infty, \delta(x) \sim x^{-(1 / \beta-\alpha-r+2)}$. Therefore, $I_{1}$ exists if and only if $1 / \beta-\alpha>r-1$. Since $\alpha>1$, this implies $1 / \beta>r$. Now, if $\alpha<1$, the inequality in (29) implies that

$$
I_{1} \leq \frac{1}{\pi \beta^{\alpha} \Gamma(\alpha)} \int_{1}^{\infty} \frac{x^{r}}{1+x^{2}}\left(1 / 2+\pi^{-1} \tan ^{-1}(x)\right)^{\alpha-1}\left(1 / 2-\pi^{-1} \tan ^{-1}(x)\right)^{1 / \beta-1} .
$$

Let $\zeta(x)=\frac{x^{r}}{1+x^{2}}\left(1 / 2+\pi^{-1} \tan ^{-1}(x)\right)^{\alpha-1}\left(1 / 2-\pi^{-1} \tan ^{-1}(x)\right)^{1 / \beta-1}$. As $x \rightarrow \infty, \zeta(x) \sim x^{-(1 / \beta-r+1)}$. So $I_{1}$ exists if and only if $1 / \beta>r$. Similarly one can show that $I_{2}$ exists if and only if $\alpha>r$.

Next, we consider recursive relation for the $r$-th moment of the $G C(\alpha, \beta, \theta)$ distribution.

Proposition 6: Let $X \sim G C(\alpha, \beta, 1)$ and $n \in \mathbb{N}$. Then

(i) $\mu_{2 n}^{\prime}(\alpha, \beta)=\frac{1}{\pi \beta(1-\beta)^{\alpha-1}} \sum_{j=1}^{n} \frac{(-1)^{j-1}}{2 n-2 j+1}\left\{\mu_{2 n-2 j+1}^{\prime}\left(\alpha, \frac{\beta}{1-\beta}\right)-\mu_{2 n-2 j+1}^{\prime}\left(\alpha-1, \frac{\beta}{1-\beta}\right)\right\}+(-1)^{n}$.

(ii) $\mu_{2 n+1}{ }^{\prime}(\alpha, \beta)=\frac{1}{\pi \beta(1-\beta)^{\alpha-1}} \sum_{j=1}^{n} \sum_{i=0}^{j} \frac{(-1)^{n-j}}{2 j}\left(\begin{array}{c}n \\ j\end{array}\right)\left(\begin{array}{l}j \\ i\end{array}\right)\left\{\mu_{2 i}^{\prime}\left(\alpha, \frac{\beta}{1-\beta}\right)-\mu_{2 i}^{\prime}\left(\alpha-1, \frac{\beta}{1-\beta}\right)\right\}+(-1)^{n} \mu^{\prime}(\alpha, \beta)$.

Proof: From (25) and using the substitution $u=\tan ^{-1}(x)$, we have

$$
\pi \beta^{\alpha} \Gamma(\alpha) \mu_{2 n}^{\prime}=\int_{-\pi / 2}^{\pi / 2}(\tan u)^{2 n}\left(-\log \left(0.5-\pi^{-1} u\right)\right)^{\alpha-1}\left(0.5-\pi^{-1} u\right)^{1 / \beta-1} d u=\sum_{j=0}^{n} I_{j},
$$

where

$$
I_{0}=(-1)^{n} \int_{-\pi / 2}^{\pi / 2}\left(-\log \left(0.5-\pi^{-1} u\right)\right)^{\alpha-1}\left(0.5-\pi^{-1} u\right)^{1 / \beta-1} d u
$$

and

$$
I_{j}=(-1)^{j-1} \int_{-\pi / 2}^{\pi / 2} \tan ^{2 n-2 j}(u) \sec ^{2}(u)\left(-\log \left(0.5-\pi^{-1} u\right)\right)^{\alpha-1}\left(0.5-\pi^{-1} u\right)^{1 / \beta-1} d u, \quad j \geq 1 .
$$

It is easy to see that $I_{0}=(-1)^{n} \pi \beta^{\alpha} \Gamma(\alpha)$. Also, it is not difficult to show that

$$
I_{j}=\frac{(-1)^{j-1} \Gamma(\alpha)}{2 n-2 j+1}\left(\frac{\beta}{1-\beta}\right)^{\alpha-1}\left\{\mu_{2 n-2 j+1}^{\prime}\left(\alpha, \frac{\beta}{1-\beta}\right)-\mu_{2 n-2 i+1}^{\prime}\left(\alpha-1, \frac{\beta}{1-\beta}\right)\right\} .
$$


Substituting (31) in (30), we get the result in (i). For the proof of (ii), one can easily see that

$$
\pi \beta^{\alpha} \Gamma(\alpha) \mu_{2 n+1}^{\prime}=(-1)^{n} \pi \beta^{\alpha} \Gamma(\alpha) \mu^{\prime}(\alpha, \beta)+\sum_{j=1}^{n} I_{j},
$$

where

$$
I_{j}=(-1)^{n-j}\left(\begin{array}{c}
n \\
j
\end{array}\right) \int_{-\pi / 2}^{\pi / 2} \sec ^{2 j-1}(u) \sec u \tan u\left(-\log \left(0.5-\pi^{-1} u\right)\right)^{\alpha-1}\left(0.5-\pi^{-1} u\right)^{1 / \beta-1} d u .
$$

The rest of the proof is not difficult to show.

Table 2 provides the mean, variance, skewness and kurtosis of the $G C(\alpha, \beta, 1)$ for various values of $\alpha$ and $\beta$. For fixed $\alpha$, the mean and skewness are increasing functions of $\beta$. Also, for fixed $\beta$, the mean is an increasing function of $\alpha$. Furthermore, the values of the skewness from Table 2 show that the distribution is very flexible in terms of shapes and the distribution can be left or right skewed.

Skewness and kurtosis of a distribution can be measured by $\beta_{1}=\mu_{3} / \sigma^{3}$ and $\beta_{2}=\mu_{4} / \sigma^{4}$, respectively. However, the third and fourth moments of $G C(\alpha, \beta, \theta)$ do not always exist (see Proposition 5). Alternatively, we can define the measure of asymmetry and tail weight based on quantile function. The Galton's skewness $S$ defined by Galton (1883) and the Moors' kurtosis $K$ defined by Moors (1988) are given by

$$
\begin{aligned}
& S=\frac{Q(6 / 8)-2 Q(4 / 8)+Q(2 / 4)}{Q(6 / 8)-Q(2 / 8)} . \\
& K=\frac{Q(7 / 8)-Q(5 / 8)+Q(3 / 8)-Q(1 / 8)}{Q(6 / 8)-Q(2 / 8)} .
\end{aligned}
$$

Table 2 Mean, variance, skewness and kurtosis calculations for $G C(\alpha, \beta, 1)$

\begin{tabular}{lllccl}
\hline$a$ & $\beta$ & Mean & Variance & Skewness & Kurtosis \\
\hline 4 & 0.01 & -10.7296 & 56.4986 & -5.2367 & $*$ \\
& 0.1 & -0.8567 & 0.7824 & -3.8549 & $*$ \\
& 0.2 & 0.0774 & 0.6096 & 0.2118 & $*$ \\
5 & 0.3 & 0.8738 & 2.7446 & 18.0382 & $*$ \\
& 0.01 & -8.0673 & 21.2643 & -3.4321 & 44.2181 \\
& 0.1 & -0.5021 & 0.3953 & -1.7868 & 17.6345 \\
& 0.2 & 0.4480 & 0.6855 & 2.4384 & 56.5459 \\
& 0.3 & 1.5732 & 6.8542 & 22.1028 & $*$ \\
& 0.01 & -4.6280 & 3.5337 & -1.9178 & 5.9542 \\
& 0.1 & 0.1248 & 0.2153 & 0.7560 & 3.2951 \\
& 0.2 & 1.6343 & 2.7528 & 6.3891 & 395.2955 \\
& 0.3 & 5.3887 & 124.9174 & 13.2288 & $*$ \\
& 0.01 & -3.5986 & 1.6310 & -1.5609 & 15.6352 \\
& 0.1 & 0.4431 & 0.2448 & 0.9704 & 8.1938 \\
& 0.2 & 2.7883 & 8.3416 & 8.3283 & 802.9949 \\
& 0.3 & 11.1914 & 5.1306 & $*$ \\
\hline
\end{tabular}


When the distribution is symmetric, $S=0$ and when the distribution is right (or left) skewed $S>0$ (or $S<0$ ). As $K$ increases the tail of the distribution becomes heavier. To investigate the effect of the two shape parameters $\alpha$ and $\beta$ on the $G C(\alpha, \beta, \theta)$ distribution, Eqs. (32) and (33) are used to obtain the Galtons' skewness and Moors' kurtosis where the quantile function is defined in Remark (ii). Figure 4 displays the Galton's skewness and Moors' kurtosis for the $G C(\alpha, \beta, 1)$. From this figure, it appears that the $G C(\alpha, \beta, \theta)$ distribution has a wide range of skewness and kurtosis. It can be left skewed, right skewed or symmetric.

\subsection{Estimation of parameters by maximum likelihood method}

Let $X_{1}, X_{2}, \ldots, X_{n}$ be a random sample of size $n$ drawn from the $G C(\alpha, \beta, \theta)$. The loglikelihood function is given by

$$
\ell=-n \log \left(\pi \theta \Gamma(\alpha) \beta^{\alpha}\right)-\log \left(1+\left(x_{i} / \theta\right)\right)+\left(\beta^{-1}-1\right) \sum_{i=1}^{n} \log \left(z_{i}\right)+(\alpha-1) \sum_{i=1}^{n} \log \left(-\log \left(z_{i}\right)\right),
$$

where $z_{i}=0.5-\pi^{-1} \tan ^{-1}\left(x_{i} / \theta\right)$.

The derivatives of (34) with respect to $\alpha, \beta$ and $\theta$ are given by

$$
\begin{aligned}
& \frac{\partial \ell}{\partial \alpha}=-n \psi(\alpha)-n \log \beta+\sum_{i=1}^{n} \log \left(-\log \left(z_{i}\right)\right), \\
& \frac{\partial \ell}{\partial \beta}=-\frac{n \alpha}{\beta}+\frac{1}{\beta^{2}} \sum_{i=1}^{n} \log \left(z_{i}\right), \\
& \frac{\partial \ell}{\partial \theta}=-\frac{n}{\theta}+\sum_{i=1}^{n} \frac{x_{i}}{\theta\left(\theta+x_{i}\right)}+\sum_{i=1}^{n} \frac{x_{i}}{\pi\left(\theta^{2}+x_{i}^{2}\right) z_{i}}\left\{(\alpha-1)\left(\log \left(z_{i}\right)\right)^{-1}+1 / \beta-1\right\},
\end{aligned}
$$

where $\psi(\alpha)=\partial \log \Gamma(\alpha) / \partial \alpha$, is the digamma function.

Therefore, the MLE $\hat{\alpha}, \hat{\beta}$ and $\theta$ are obtained by setting the Eqs. (35), (36) and (37) to zero and solving them simultaneously. Note that the number of equations can be reduced to two by using Eq. (36) to get $\beta=\sum_{i=1}^{n} \frac{\log \left(z_{i}\right)}{n \alpha}$. The initial value for the
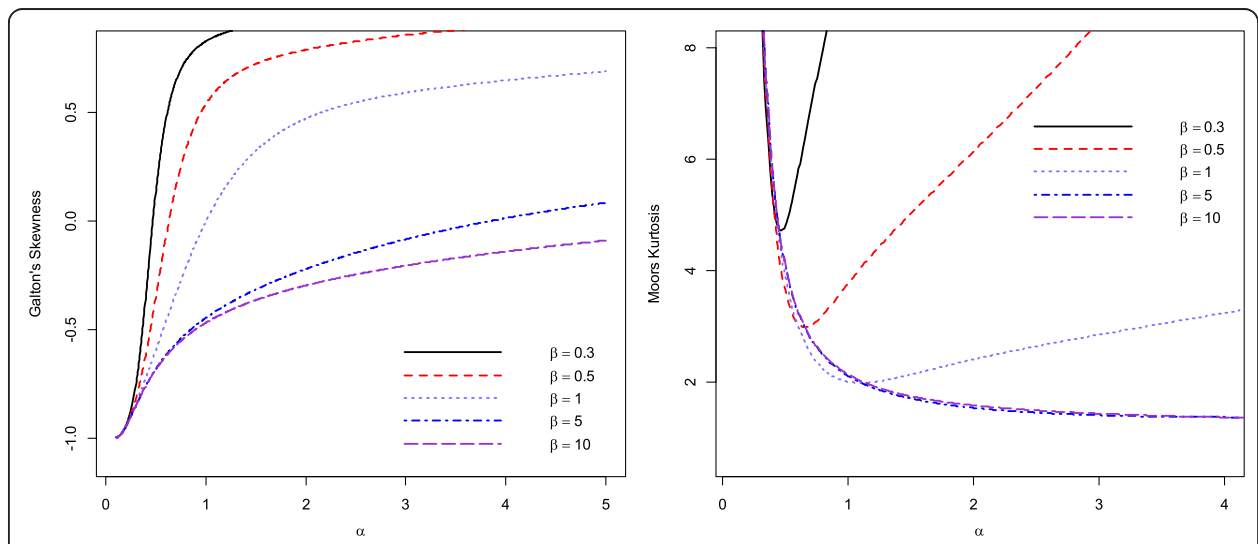

Fig. 4 Graphs of Galton's skewness and Moors' kurtosis for the distribution $G C(\alpha, \beta, 1)$ 
parameter $\theta$ can be taken as $\theta_{0}=1$. From Remark (i) in Gamma-Cauchy\{exponential\} distribution, the random variable $Y_{i}=-\log \left[0.5-\pi^{-1} \tan ^{-1}\left(X_{i} / \theta_{0}\right)\right], i=1,2, \ldots, n$ follows a gamma distribution with parameters $\alpha$ and $\beta$. Therefore, by equating the sample mean and sample variance of $Y_{i}$ with the corresponding population mean and variance, the initial estimates for $\alpha$ and $\beta$ are, respectively, $\alpha_{0}=\bar{y}^{2} / s_{y}^{2}$ and $\beta_{0}=s_{y}^{2} / \bar{y}$ where $\bar{y}$ and $s_{y}^{2}$ are the sample mean and sample variance for $y_{i}, i=1, \ldots, n$.

\subsection{Simulation study}

A simulation study is conducted to evaluate the MLE in terms of estimates and standard deviations for various parameter combinations and different sample sizes. We consider the values $0.5,0.9,1,2,5$ for the parameter $\alpha, 0.5,1,3$ for the parameter $\beta$, and 1 , 2 for the parameter $\theta$. The simulation study for the MLE is conducted for a total of six parameter combinations and the process is repeated 200 times. Three different sample sizes $n=50,100,300$ are considered. The ML estimates and the standard deviations are presented in Table 3. From this table, it appears that the ML estimates of $\alpha$ and $\theta$ tend to be overestimated. As expected, as the sample size increases, the bias and standard deviation values for all the estimates decrease.

\subsection{Applications}

In this section, the $G C(\alpha, \beta, \theta)$ distribution is fitted to two data sets. The first data set from Bjerkedal (1960), represents the survival time in days of 72 guinea pigs infected with virulent tubercle bacilli. The first data set is

$10,33,44,56,59,72,74,77,92,93,96,100,100,102,105,107,107,108,108,108,109,112,121,122,122,124,130$ $134,136,139,144,146,153,159,160,163,163,168,171,172,176,113,115,116,120,183,195,196,197,202,213$, $215,216,222,230,231,240,245,251,253,254,255,278,293,327,342,347,361,402,432,458,555$

The data is skewed-to-the right with skewness $=1.3134$ and kurtosis $=3.8509$.

The second data set from Durbin and Koopman (2001), represents the measurements of the annual flow of the Nile River at Ashwan from 1871-1970. The second data set is

$1120,1160,963,1210,1160,1160,813,1230,1370,1140,995,935,1110,994,1020,960,1180,799,958,1140$ $1100,1210,1150,1250,1260,1220,1030,1100,774,840,874,694,940,833,701,916,692,1020,1050,969$, $831,726,456,824,702,1120,1100,832,764,821,768,845,864,862,698,845,744,796,1040,759,781,865$, $845,944,984,897,822,1010,771,676,649,846,812,742,801,1040,860,874,848,890,744,749,838,1050$, $918,986,797,923,975,815,1020,906,901,1170,912,746,919,718,714,740$

The data is approximately symmetric with skewness $=0.3175$ and kurtosis $=2.6415$.

We fitted the two data sets to the $G C(\alpha, \beta, \theta)$ distribution and compared the results with Cauchy, gamma-Pareto proposed by Alzaatreh et al. (2012) and betaCauchy distributions proposed by Alshawarbeh et al. (2013). The maximum likelihood estimates, the log-likelihood value, the AIC (Akaike Information Criterion), the Kolmogorov-Smirnov (K-S) test statistic, and the $p$-value for the K-S statistic for the fitted distributions to the data sets 1 and 2 are reported in Tables 4 and 5 respectively.

The results in Tables 4 and 5 show the $G C(\alpha, \beta, \theta)$ and beta-Cauchy provide an adequate fit to the survival time data while the $G C(\alpha, \beta, \theta)$ distribution provides the best 
Table 3 Estimates and standard deviations for the parameters using MLE method

\begin{tabular}{|c|c|c|c|c|c|c|c|c|c|}
\hline \multirow{2}{*}{$\begin{array}{l}\text { Sample size } \\
n\end{array}$} & \multicolumn{3}{|c|}{ Actual values } & \multicolumn{3}{|c|}{ ML estimates } & \multicolumn{3}{|c|}{ Standard deviation } \\
\hline & $a$ & $\beta$ & $\theta$ & $\hat{a}$ & $\hat{\beta}$ & $\hat{\theta}$ & $\hat{a}$ & $\hat{\beta}$ & $\hat{\theta}$ \\
\hline \multirow[t]{6}{*}{50} & 1 & 1 & 1 & 1.1605 & 0.9260 & 1.1455 & 0.0518 & 0.0460 & 0.0538 \\
\hline & 0.5 & 1 & 2 & 0.5377 & 0.9552 & 2.1399 & 0.0180 & 0.0426 & 0.0978 \\
\hline & 0.5 & 3 & 2 & 0.5351 & 2.8849 & 2.1952 & 0.0154 & 0.1207 & 0.1065 \\
\hline & 0.9 & 0.5 & 1 & 1.0229 & 0.4960 & 1.0579 & 0.0481 & 0.0222 & 0.0442 \\
\hline & 2 & 0.5 & 1 & 2.9571 & 0.4448 & 1.2255 & 0.4271 & 0.0282 & 0.0917 \\
\hline & 5 & 0.5 & 1 & 7.9697 & 0.4487 & 0.8862 & 0.9537 & 0.0183 & 0.0737 \\
\hline \multirow[t]{6}{*}{100} & 1 & 1 & 1 & 1.0717 & 0.9617 & 1.0499 & 0.0216 & 0.0230 & 0.0248 \\
\hline & 0.5 & 1 & 2 & 0.5182 & 1.0090 & 2.0843 & 0.0084 & 0.0229 & 0.0457 \\
\hline & 0.5 & 3 & 2 & 0.5165 & 2.9594 & 2.0505 & 0.0065 & 0.0591 & 0.0441 \\
\hline & 0.9 & 0.5 & 1 & 0.9375 & 0.4932 & 1.0309 & 0.0181 & 0.0105 & 0.0203 \\
\hline & 2 & 0.5 & 1 & 2.2258 & 0.4753 & 1.0866 & 0.0646 & 0.0131 & 0.0281 \\
\hline & 5 & 0.5 & 1 & 6.3436 & 0.4737 & 0.9243 & 0.4015 & 0.0089 & 0.0367 \\
\hline \multirow[t]{6}{*}{300} & 1 & 1 & 1 & 1.0244 & 0.9983 & 1.0270 & 0.0094 & 0.0114 & 0.0111 \\
\hline & 0.5 & 1 & 2 & 0.5076 & 0.9908 & 2.0320 & 0.0041 & 0.0102 & 0.0229 \\
\hline & 0.5 & 3 & 2 & 0.5113 & 2.9636 & 2.0718 & 0.0039 & 0.0315 & 0.0241 \\
\hline & 0.9 & 0.5 & 1 & 0.9242 & 0.4953 & 1.0159 & 0.0086 & 0.0053 & 0.0102 \\
\hline & 2 & 0.5 & 1 & 2.1286 & 0.4844 & 1.0369 & 0.0270 & 0.0071 & 0.0123 \\
\hline & 5 & 0.5 & 1 & 5.2815 & 0.4987 & 0.9562 & 0.1368 & 0.0040 & 0.0149 \\
\hline
\end{tabular}

fit (based on KS $p$-value) to the annual flow of the Nile River data. The fact that $G C(\alpha, \beta, \theta)$ distribution has only three parameters compared with the beta-Cauchy distribution makes $G C(\alpha, \beta, \theta)$ a natural choice for fitting these two data sets. A closer look at the parameter estimates for the beta-Cauchy distribution indicates that the estimates of $\alpha, \beta$ and $\theta$ in the beta-Cauchy distribution are not statistically significant for the two examples. This is an indication that beta-Cauchy is over-parameterized for fitting these two data sets. This supports the point that the three-parameter $G C(\alpha, \beta, \theta)$ distribution should be used to fit the two data sets. Figure 5 displays the histogram and the fitted density functions for the two data sets, which support the results in Tables 4 and 5.

Table 4 Parameter estimates for the survival time data

\begin{tabular}{lllll}
\hline Distribution & Cauchy & Gamma-Pareto & Beta-Cauchy & Gamma-Cauchy\{exponential\} \\
\hline Parameter & $\hat{C}=139.3079$ & $\hat{a}=6.030$ & $\hat{a}=13.9274$ & $\hat{a}=16.1591$ \\
Estimates & $(9.4281)^{\mathrm{a}}$ & $(0.9770)$ & $(18.5335)$ & $(2.6666)$ \\
& $\hat{\theta}=48.1262$ & $\hat{c}=0.4497$ & $\hat{\beta}=4.5828$ & $\hat{\beta}=0.1027$ \\
& $(7.6793)$ & $(0.0760)$ & $(3.6504)$ & $(0.0238)$ \\
& & $\hat{\theta}=10$ & $\begin{array}{l}\hat{C}=117.9055 \\
(37.6269)\end{array}$ & $\begin{array}{l}\hat{\theta}=110.1742 \\
(35.4345)\end{array}$ \\
& & & $\hat{\theta}=27.0884$ & \\
& & & $(99.7681)$ & \\
Log-likelihood & -437.5967 & -465.4670 & -424.4339 & -424.4423 \\
AlC & 879.1934 & 934.9340 & 856.8679 & 854.8847 \\
K-S & 0.1416 & 0.2606 & 0.0760 & 0.0752 \\
K-S p-value & 0.1114 & 0.0001 & 0.8005 & 0.8105 \\
\hline
\end{tabular}

${ }^{a_{\text {standard }}}$ error 
Table 5 Parameter estimates for the annual flow of the Nile River data

\begin{tabular}{lllll}
\hline Distribution & Cauchy & Gamma-Pareto & Beta-Cauchy & Gamma-Cauchy\{exponential\} \\
\hline Parameter & $\hat{C}=879.3679$ & $\hat{a}=5.0437$ & $\hat{a}=50.9201$ & $\hat{a}=322.5715$ \\
Estimates & $(17.3969)^{\mathrm{a}}$ & $(0.6902)$ & $(66.0939)$ & $(6.4901)$ \\
& $\hat{\theta}=103.8804$ & $\hat{C}=0.1357$ & $\hat{\beta}=25.1275$ & $\hat{\beta}=0.0103$ \\
& $(13.44841)$ & $(0.0195)$ & $\begin{array}{l}(29.7478) \\
(\hat{C}=712.2062\end{array}$ & $\begin{array}{l}\hat{\theta}=10003) \\
(445.6252)\end{array}$ \\
& & $\hat{\theta}=456$ & $\begin{array}{l}\hat{\theta}=48.5797 \\
(361.7110)\end{array}$ & \\
& & & -653.4892 & -654.3825 \\
Log-likelihood & -674.4637 & -696.7975 & 1314.9780 & 1314.7650 \\
AIC & 1352.9270 & 1397.5950 & 0.0736 & 0.0637 \\
K-S & 0.1311 & 0.1705 & 0.6515 & 0.8120 \\
K-S p-value & 0.0642 & 0.0060 & & \\
\hline
\end{tabular}

${ }^{\mathrm{a}}$ standard error

\section{Concluding remarks}

A family of generalized Cauchy distributions, $T$-Cauchy $\{Y\}$ family, is proposed using the $T-R\{Y\}$ framework. Several properties of the $T$-Cauchy $\{Y\}$ family are studied including moments and Shannon's entropy. Some members of the T-Cauchy $\{Y\}$ family are presented. A member of the $T$-Cauchy $\{Y\}$ family, the gammaCauchy\{exponential\} distribution, is studied in detail. This distribution is interesting as it consists of exponentiated Cauchy distribution and distributions of record values of Cauchy distribution as special cases. Various properties of the gammaCauchy\{exponential\} distribution are studied, including mode, moments and Shannon's entropy. Unlike the Cauchy distribution, the gamma-Cauchy\{exponential\} distribution can be right-skewed or left-skewed. Also, the moments of the gammaCauchy\{exponential\} distribution exist under certain restrictions on the parameters. In particular, the $r$-th moment for the gamma- Cauchy\{exponential\} distribution exists if and only if $\alpha, \beta^{-1}>r$ and this is not the case for the Cauchy distribution. The flexibility of the gamma-Cauchy\{exponential\} distribution and the existence of the moments in some cases make this distribution as an alternate to the Cauchy distribution in situations where the Cauchy distribution may not provide an adequate fit.
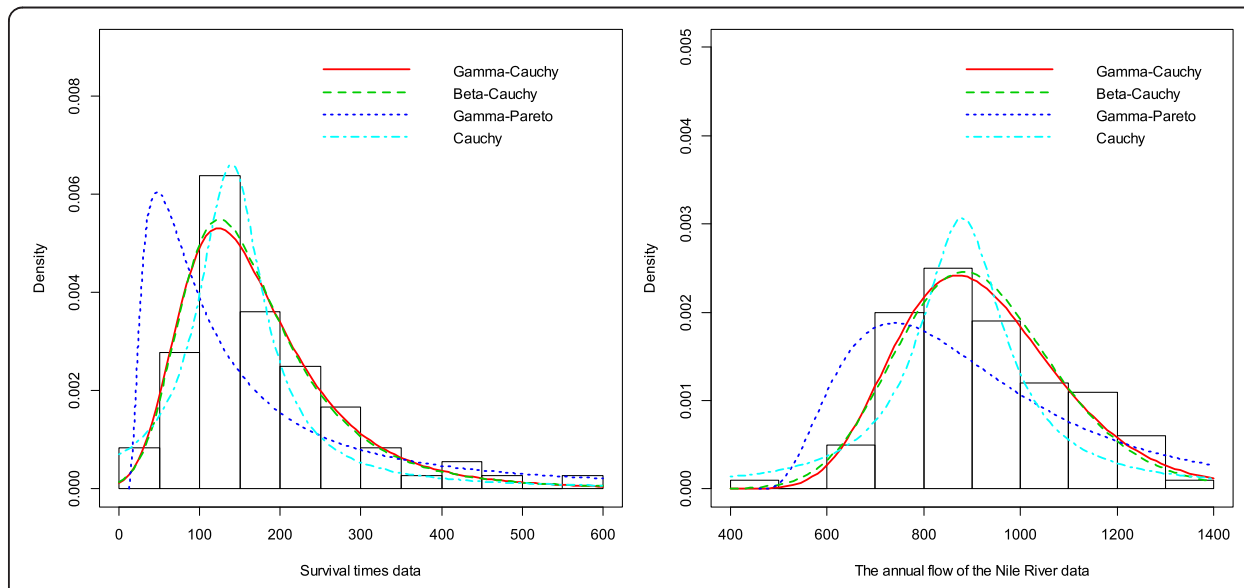

Fig. 5 Histograms and the fitted distributions for the two data sets 
Acknowledgement

The first author gratefully acknowledges the support received from the Social Fund Policy Grant at Nazarbayev University.

\section{Authors' contributions}

The authors, AA, CL, FF and IG with the consultation of each other carried out this work and drafted the manuscript together. All authors read and approved the final manuscript.

\section{Competing interests}

The authors declare that they have no competing interests.

\section{Author details}

'Department of Mathematics, Nazarbayev University, Astana, KZ 010000, Kazakhstan. '²epartment of Mathematics, Central Michigan University, Mount Pleasant, Ml 48859, USA. ${ }^{3}$ Department of Mathematics and Statistics, University of North Carolina Wilmington, Wilmington, NC 28403, USA.

Received: 23 February 2016 Accepted: 13 July 2016

Published online: 03 August 2016

\section{References}

Abramowitz, M, Stegun, IA: Handbook of mathematical functions with formulas, graphs and mathematical tables, vol. 55. Dover Publication, Inc., New York (1964)

Aljarrah, MA, Lee, C, Famoye, F: On generating T-X family of distributions using quantile functions. J. Stat. Distrib. Appl. $1,1-17$ (2014)

Almheidat, M, Famoye, F, Lee, C: Some generalized families of Weibull distribution: properties and applications. Int. J. Stat. Probab. 4, 18-35 (2015)

Alshawarbeh, E, Lee, C, Famoye, F: The beta-Cauchy distribution. J. Probab. Stat. Sci. 10(1), 41-57 (2012)

Alshawarbeh, E, Famoye, F, Lee, C: Beta-Cauchy distribution: some properties and applications. J. Stat. Theory Appl. 12(4), 378-391 (2013)

Alzaatreh, A, Famoye, F, Lee, C: Gamma-Pareto distribution and its applications. J. Mod. Appl. Stat. Methods 11(1), 78-94 (2012)

Alzaatreh, A, Lee, C, Famoye, F: A new method for generating families of continuous distributions. Metron 71(1), 63-79 (2013)

Alzaatreh, A, Lee, C, Famoye, F: T-normal family of distributions: a new approach to generalize the normal distribution. J. Stat. Distrib. Appl. 1, 1-16 (2014)

Alzaatreh, A, Lee, C, Famoye, F: Family of generalized gamma distributions: properties and applications. To appear in Hacettepe Journal of Mathematics and Statistics. (2015)

Bjerkedal, T: Acquisition of resistance in guinea pigs infected with different doses of virulent tubercle bacilli. Am. J. Epidemiol. 72, 130-148 (1960)

Durbin, J, Koopman, SJ: Time series analysis by state space methods. Oxford University Press, Oxford (2001)

Eugene, N, Lee, C, Famoye, F: Beta-normal distribution and its applications. Commun. Stat. Theory Methods 31(4), 497$512(2002)$

Galton, F: Enquiries into human faculty and its development. Macmillan, London (1883)

Gradshteyn, IS, Ryzhik, IM: Tables of integrals, series and products, 7th edn. Elsevier, Inc., London (2007)

Johnson, NL, Kotz, S, Balakrishnan, N: Continuous Univariate Distributions, vol. 1, 2nd edn. Wiley, New York (1994)

Moors, JJA: A quantile alternative for kurtosis. Statistician 37, 25-32 (1988)

Sarabia, JM. Castillo, E: About a class of max-stable families with applications to income distributions. Metron 63, 505-527 (2005)

Shannon, CE: A mathematical theory of communication. Bell Syst. Tech. J. 27, 379-432 (1948)

Stigler, SM: Letter to the editor: normal orthant probabilities. Am. Stat. 43(4), 291 (1989)

\section{Submit your manuscript to a SpringerOpen ${ }^{\circ}$ journal and benefit from:}

- Convenient online submission

- Rigorous peer review

- Immediate publication on acceptance

- Open access: articles freely available online

- High visibility within the field

- Retaining the copyright to your article 